\title{
A note on the noncommutative correction to gravity
}

\author{
Pradip Mukherjee* a,b Anirban Saha ${ }^{\text {a,c }}$ \\ ${ }^{a}$ Department of Physics, Presidency College \\ 86/1 College Street, Kolkata - 700 073, India \\ ${ }^{\mathrm{b}}$ pradip@bose.res.in and \\ ${ }^{c}$ ani_saha09@dataone.in
}

\begin{abstract}
An apparent contradiction in the leading order correction to noncommutative (NC) gravity reported in the literature has been pointed out. We show by direct computation that actually there is no such controvarsy and all perturbative NC corrections start from the second order in the NC parameter. The role of symmetries in the vanishing of the first order correction is manifest in our calculation.
\end{abstract}

PACS numbers: $11.10 . \mathrm{Nx}, 04.20 .-\mathrm{q}$

\section{INTRODUCTION}

The idea of fuzzy space time where the coordinates $x^{\mu}$ satisfy the noncommutative (NC) algebra

$$
\left[x^{\mu}, x^{\nu}\right]=i \theta^{\mu \nu}
$$

with constant anti-symmetric $\theta^{\mu \nu}$, was mooted long ago 1 . This idea has been revived in the recent past and field theories defined over this NC space has been studied extensively ${ }^{2}$. As always, a colossal challenge in this context comes from general theory of relativity (GTR). There are various attempts to fit GTR in the context of NC space time. Broadly there are two different approaches to the analysis of $\mathrm{NC}$ theories. One approach is to treat the fields as operators in some Hilbert space. The other approach is to define the fields over phase space with ordinary multiplication replaced by the GronewaldMoyal product. In the later approach the original theory can be mapped to an equivalent commutative theory in the framework of perturbative expansion in the NC parameter, using the Seiberg-Witten-type maps ${ }^{3.4}$ for the fields. This commutative equivalent approach has been used to analyse many gauge theories in the recent past $t^{5}$. Since gravity can be viewed effectively as a gauge theory the commutative equivalent approach seems to be a promising one. Indeed, a minimal theory of $\mathrm{NC}$ gravity $\underline{6}$ has been constructed recently based on this approach where the $\mathrm{NC}$ correction appears as a series expansion in the NC parameter. The leading order correction is reported to be linear in $\theta$ in this work.

Construction of a theory of NC gravity remains a topic of considerable current interest in the literature and various authors have approached the problem from different angles. $\mathrm{In}^{7}$ for example a deformation of Einstein's gravity was studied using a construction based on gauging

\footnotetext{
*Also Visiting Associate, S. N. Bose National Centre for Basic Sciences, JD Block, Sector III, Salt Lake City, Calcutta -700 098, India and

IUCAA, Post Bag 4, Pune University Campus, Ganeshkhind, Pune 411 007,India
}

the noncommutative $\mathrm{SO}(4,1)$ de Sitter group and the SW $\mathrm{map}^{3}$ with subsequent contraction to $\operatorname{ISO}(3,1)$. Another construction of a noncommutative gravitational theory was proposed in ${ }^{8}$. Very recently noncommutative gravity has been connected with stringy perspective $e^{\frac{9}{}}$. In all these works the leading order noncommutative effects appear in the second order in the NC parameter $\theta$. It seems thus that the result of $\mathrm{f}^{6}$ is in contradiction. In this brief report we extend their work to show that actually there is no such controversy.

The organisation of this report is as follows. In the next section we present a brief review of the results of f $^{6}$. This will be useful as the starting point of our calculation as well as to fix the notations. In section 3 details of computation of the first order correction term has been given. Section 4 contains the concluding remarks.

\section{REVIEW OF MINIMAL FORMULATION OF NC GRAVITY}

The main problem of implementing GTR on NC platform is that the algebra (11) is not invariant under general coordinate transformation. However, we can identify a subclass of general coordinate transformations,

$$
\hat{x}^{\mu \prime}=\hat{x}^{\mu}+\hat{\xi}^{\mu}(\hat{x}),
$$

which are compatible with the algebra given by (11). This imposes a restriction on $\xi^{\mu}$

$$
\theta^{\mu \alpha} \hat{\partial}_{\alpha} \hat{\xi}^{\nu}(\hat{x})=\theta^{\nu \beta} \hat{\partial}_{\beta} \hat{\xi}^{\mu}(\hat{x}) .
$$

and the theory corresponds to the version of General Relativity based on volume-preserving diffeomorphism known as the unimodular theory of gravitation ${ }^{10}$. Thus the symmetries of canonical noncommutative space time naturally lead to the noncommutative version of unimodular gravity ${ }^{6}$. With the symmetries preserved in this manner the extension of GTR to noncommutative perspective is done using the tetrad formalism and invoking the enveloping algebra method ${ }^{11}$. The theory is then cast in the commutative equivalent form by the use of appropriate Seiberg-Witten (SW) maps. The final form of NC 
action is

$$
S=\int d^{4} x \frac{1}{2 \kappa^{2}} \hat{R}(\hat{x})
$$

$\hat{R}$ is the noncommutative version of the Ricci scalar

$$
\hat{R}=\hat{R}_{a b}^{a b}
$$

where $\hat{R}_{a b}{ }^{c d}$ are the components of the NC Riemann tensor appearing in

$$
\hat{R}_{a b}(\hat{x})=\frac{1}{2} \hat{R}_{a b}{ }^{c d}(\hat{x}) \Sigma_{c d}
$$

The latin indices refer to the vierbein and $\Sigma_{c d}$ are the generators of the local Lorentz algebra $S O(3,1)$.

$\hat{R}_{a b}(\hat{x})$ can be expanded as 6

$$
\hat{R}_{a b}=R_{a b}+R_{a b}^{(1)}+\mathcal{O}\left(\theta^{2}\right)
$$

with

$$
R_{a b}^{(1)}=\frac{1}{2} \theta^{c d}\left\{R_{a c}, R_{b d}\right\}-\frac{1}{4} \theta^{c d}\left\{\omega_{c},\left(\partial_{d}+D_{d}\right) R_{a b}\right\} .
$$

In the above expression $\omega_{a}^{b c}$ are the spin connection fields

$$
\omega_{a}(x)=\frac{1}{2} \omega_{a}^{b c} \Sigma_{b c}
$$

which are antisymmetric under the exchange of $b$ and $c$. Again $D_{a}$ is the covariant derivative

$$
D_{a}=\partial_{a}+\frac{i}{2} \omega_{a}^{b c} \Sigma_{b c}
$$

Note that all quantities appearing on the rhs of (8) are ordinary commutative functions.

Using the above expansion we can write from (4)

$$
S=\int d^{4} x \frac{1}{2 \kappa^{2}}\left(R(x)+R^{(1)}(x)\right)+\mathcal{O}\left(\theta^{2}\right) .
$$

$R(x)$ is the usual Ricci scalar and $R^{(1)}(x)$ is its first order correction. In the following section we will explicitly compute this correction term .

\section{EXPLICIT COMPUTATION OF THE FIRST ORDER CORRECTION TERM}

For the computation of the first order term we need an explicit form of $\Sigma_{c d}$, the generators of the local Lorentz algebra $S O(3,1)$. This is given by ${ }^{12}$

$$
\left[\Sigma_{c d}\right]_{b}^{a}=\delta^{a}{ }_{c} \eta_{d b}-\delta^{a}{ }_{d} \eta_{c b}
$$

where $\eta_{a b}=\operatorname{diag}(-,+,+,+)$. As stated, the latin indices refer to the vierbein. Our plan of calculation is as follows. Using (12) we compute the first order correction to the Ricci tensor (8). Since by finding the $a b$ component of $\hat{R}_{a b}$ we get $\hat{R}$ on contraction, the corresponding first order correction to the NC action can now be calculated.

We now proceed to compute the correction term $R^{(1)}(x)$. First note that $R^{(1)}(x)=R_{a b}^{(1) a b}$. From (6) and (7) this is equal to $\left[R_{a b}^{(1)}\right]^{a b}$. We thus have to calculate the corresponding matrix element of the rhs of (8) and contract. For convenience we write the result as

$$
\left[R_{a b}^{(1)}\right]^{a b}=T_{1}+T_{2}
$$

where $T_{1}$ and $T_{2}$ denote respectively the contributions coming from the first and the second terms. Now after some computation we get

$$
T_{1}=2 \theta^{c d}\left[R_{a c g}{ }^{a} R_{b d}^{b g}+R_{a c}^{b}{ }^{b} R_{b d}{ }^{g a}\right]
$$

The computation of the second term is somewhat involved. We first compute the part containing the covariant derivative as

$$
\left[\left(\partial_{d}+D_{d}\right) R_{a b}\right]_{f}^{e}=2 \partial_{d} R_{a b}{ }_{f}+i \omega_{d}^{e g} R_{a b g f}
$$

Using this expression for the derivative term we compute the second term to be

$$
\begin{aligned}
T_{2}=-\theta^{c d} & {\left[\frac{1}{2}\left(\omega_{c}{ }^{a j} \partial_{d} R_{a b j}{ }^{b}-\omega_{c}{ }^{a j} \partial_{d} R_{b a}{ }^{b}{ }_{j}\right)\right.} \\
+ & \left.\frac{i}{4}\left(\omega_{c}{ }^{a j} \omega_{d j}{ }^{g} R_{a b g}{ }^{b}-\omega_{d}{ }^{a j} \omega_{c}^{b g} R_{a b j g}\right)\right]
\end{aligned}
$$

One can easily see that the first two terms cancel remembering the fact that the indices of the Riemann tensor refer to the tetrad and hence raised or lowered by $\eta_{a b}$. Collecting all the nonvanishing terms from (14 16) we get the correction term $R^{(1)}(x)$ as

$$
\begin{aligned}
R^{(1)}(x)= & \theta^{c d}\left[2\left(R_{a c g}{ }^{a} R_{b d}{ }^{b g}+R_{a c}{ }^{b}{ }_{g} R_{b d}{ }^{g a}\right)\right. \\
& \left.-\frac{i}{4}\left(\omega_{c}{ }^{a j} \omega_{d j}{ }^{g} R_{a b g}{ }^{b}-\omega_{d}{ }^{a j} \omega_{c}{ }^{b g} R_{a b j g}\right)\right](17
\end{aligned}
$$

This concludes our computation. Now one can show that all the terms of the above equation (17) individually vanishes exploiting the antisymmetry of $\theta^{a b}$ and the various symmetry properties of the Riemann tensor and the spin connection fields.

\section{CONCLUSION}

Formulation of gravity in the perspective of noncommutative (NC) space time remains a topic of considerable current interest. There is an apparent contradiction in the results reported in the literature in the sense that 
where as in most of the works the noncommutative correction starts from the second order ${ }^{7.8 .9}$, a minimal formulation of $\mathrm{NC}$ gravity ${ }^{6}$ reports a first order correction. We have explicitly computed the first order correction term of the later work and demonstrated that it vanishes. This has been shown to be due to the symmetries of the various factors involved in the correction term. It appears that in the perturbative framework the order $\theta$ correction must vanish because the zero order theory carries full local Lorentz symmetry.

\section{ACKNOWLEDGEMENT}

AS would like to thank the Council for Scientific and Industrial Research (CSIR), Govt. of India, for financial support. The authors also acknowledge the hospitality of IUCAA where part of the work has been done.

PS After the submission of our comment to the Physical review D there appeared a paper ${ }^{13}$ by the authors of 6 where they have given results of calculation to the second order. Naturally they have also found that the first order correction vanishes.
${ }^{1}$ Heisenberg first suggested this idea which was later developed by Snyder; H. S. Snyder, Phys. Rev. 71 (1947) 38; ibid 72 (1947) 874.

2 See R. J. Szabo, Phys. Rep. 378 (2003) 207 and the references therein.

3 N. Seiberg, E. Witten, JHEP 9909, 032 (1999) hep-th/9908142.

4 A. A. Bichl, J. M. Grimstrup, L. Popp, M. Schweda, R. Wulkenhaar, hep-th/0102103.

5 O. F. Dayi, B. Yapiskann, JHEP 10 (2002) 022, hep-th/0208043; S. Ghosh, Nucl.Phys. B 670 (2003) 359, hep-th/0306045; B. Chakraborty, S. Gangopadhyay, A. Saha, Phys. Rev. D 70 (2004) 107707, hep-th/0312292; S. Ghosh, Phys.Rev.D70 (2004) 085007, hep-th/0402029; P. Mukherjee, A. Saha, Mod.Phys.Lett.A21 (2006) 821, hep-th/0409248; P. Mukherjee, A. Saha, hep-th/0605123; A. Saha, A. Rahaman, P. Mukherjee, hep-th/0603050.

6 X. Calmet, A. Kobakhidze, Phys. Rev. D 72 045010,2005, hep-th/0506157
7 A. H. Chamseddine, Phys. Lett. B 504, 33 (2001) hep-th/0009153.

8 P. Aschieri, C. Blohmann, M. Dimitrijevic, F. Meyer, P. Schupp, J. Wess Class.Quant.Grav. 223511 (2005), hep-th/0504183

9 L. Alvarez-Gaume, F. Meyer, M. A. Vazquez-Mozo, hep-th/0605113 ]

10 J. J. van der Bij, H. van Dam and Y. J. Ng, Physica 116A, 307 (1982), F. Wilczek, Phys. Rept. 104, 143 (1984); W. Buchmuller and N. Dragon, Phys. Lett. B 207, 292 (1988); M. Henneaux and C. Teitelboim,Phys. Lett. B 222, 195 (1989); W. G. Unruh, Phys. Rev. D 40, 1048 (1989).

11 B. Jurco, S. Schraml, P. Schupp and J. Wess, Eur. Phys. J. C 17, 521 (2000), hep-th/0006246.

12 S. Weinberg, Gravitation and Cosmology: Principles and Applications opf the General Theory of Relativity, John Wiley \& Sons. , New York, 1972.

13 X. Calmet, A. Kobakhidze, hep-th/0605275 